\title{
Qualitative and quantitative comparison of image quality between single-shot echo-planar and interleaved multi-shot echo-planar diffusion-weighted imaging in female pelvis
}

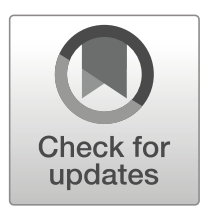

\author{
He $\mathrm{An}^{1}$ (1) $\cdot$ Xiaodong $\mathrm{Ma}^{2}$ (D) $\cdot{\text { Ziyi } \operatorname{Pan}^{2} \text { (D) Hua Guo }}^{2}$ (D) Elaine Yuen Phin Lee ${ }^{1}$ (D)
}

Received: 11 July 2019 / Revised: 20 August 2019 / Accepted: 2 October 2019 / Published online: 10 December 2019

(C) The Author(s) 2019

\begin{abstract} shot echo-planar (IMS-EPI) diffusion-weighted imaging (DWI) in female pelvis and quantitative parameters were compared with paired $t$ test and Bland-Altman analysis. two sequences. though the SNR and CNR decreased due to post-processing limitations.

Key Points

- IMS-EPI showed better image quality than SS-EPI.

- IMS-EPI showed lower geometric distortion without affecting ADC compared with SS-EPI.

- The SNR and CNR of IMS-EPI decreased due to post-processing limitations.
\end{abstract}

Objectives To qualitatively and quantitatively compare the image quality between single-shot echo-planar (SS-EPI) and multi-

Methods This was a prospective study involving 80 females who underwent 3.0T pelvic magnetic resonance imaging (MRI). SS-EPI and IMS-EPI DWI were acquired with $3 b$ values $\left(0,400,800 \mathrm{~s} / \mathrm{mm}^{2}\right)$. Two independent reviewers assessed the overall image quality, artifacts, sharpness, and lesion conspicuity based on a 5-point Likert scale. Regions of interest (ROI) were placed on the endometrium and the gluteus muscles to quantify the signal intensities and apparent diffusion coefficient (ADC). Signalto-noise ratio (SNR), contrast-to-noise ratio $(\mathrm{CNR})$, and geometric distortion were quantified on both sequences. Inter-rater agreement was assessed using $\kappa$ statistics and Kendall test. Qualitative scores were compared using Wilcoxon signed-rank test

Results IMS-EPI demonstrated better image quality than SS-EPI for all aspects evaluated (SS-EPI vs. IMS-EPI: overall quality 3.04 vs. 4.17 , artifacts 3.09 vs. 3.99 , sharpness 2.40 vs. 4.32 , lesion conspicuity 3.20 vs. $4.25 ; p<0.001$ ). Good agreement and correlation were observed between two reviewers (SS-EPI $\kappa 0.699, r 0.742$; IMS-EPI $\kappa 0.702, r 0.789$ ). IMS-EPI showed lower geometric distortion, SNR, and CNR than SS-EPI $(p<0.050)$. There was no significant difference in the mean ADC between the

Conclusion IMS-EPI showed better image quality with lower geometric distortion without affecting the quantification of ADC,

Keywords Echo-planar imaging $\cdot$ Diffusion magnetic resonance imaging $\cdot$ Female $\cdot$ Pelvis $\cdot$ Artifacts

Hua Guo

huaguo@mail.tsinghua.edu.cn

Elaine Yuen Phin Lee

eyplee77@hku.hk

1 Department of Diagnostic Radiology, Queen Mary Hospital, University of Hong Kong, Room 406, Block K, Pok Fu Lam Road, Hong Kong, China

2 Center for Biomedical Imaging Research, Department of Biomedical Engineering, School of Medicine, Tsinghua University, Haidian District, Beijing, China

$\begin{array}{ll}\text { Abbreviations and acronyms } \\ \text { ADC } & \text { Apparent diffusion coefficient } \\ \text { CNR } & \text { Contrast-to-noise ratio } \\ \text { DWI } & \text { Diffusion-weighted imaging } \\ \text { FOV } & \text { Field of view } \\ \text { GM } & \text { Gluteus muscles } \\ \text { IMS-EPI } & \text { Interleaved multi-shot echo-planar } \\ & \text { imaging } \\ \text { MRI } & \text { Magnetic resonance imaging } \\ \text { PMI } & \text { Parametrial invasion } \\ \text { ROC } & \text { Receiver operating characteristic } \\ \text { ROI } & \text { Regions of interest } \\ \text { SNR } & \text { Signal-to-noise ratio }\end{array}$

\section{Abbreviations and acronyms}


SS-EPI Single-shot k-space trajectory echo-planar imaging

\section{Introduction}

Magnetic resonance imaging (MRI) is used in the evaluation of malignant and benign diseases of the female pelvis due to its exquisite soft tissue resolution and anatomical details. Diffusion-weighted imaging (DWI) is routinely added as part of the MRI protocol [1-3]. The DWI signal varies according to the tissue microarchitecture or cellularity, reflecting the proportion of intracellular and extracellular water molecules. The log of the slope of the signal decay on DWI is quantified by the apparent diffusion coefficient (ADC), a measure of the diffusion ability of the tissue under investigation [3].

Conventional DWI uses single-shot k-space trajectory echo-planar imaging (SS-EPI), which has the advantage of fast imaging speed and thus, less sensitive to motion [4]. However, SS-EPI can suffer from geometric distortion along tissue boundaries with different susceptibilities since it usually has low bandwidth along the phase-encoding direction [5]. Moreover, SS-EPI has a relatively long readout duration compared with the transverse relaxation time, which can result in blurring artifacts and limit spatial resolution. Therefore, SSEPI gives rise to low-resolution images and encounters difficulty with large field of view (FOV) [6].

High spatial resolution imaging is important in the assessment of gynecological tumors as a clear and undistorted tumor delineation will allow confident diagnosis and accurate evaluation of the local disease extent. However, peristalsis and air in the gastrointestinal tract and vagina exaggerate the artifacts and the geometric distortion on SS-EPI, hence challenging to achieve high spatial resolution on SS-EPI.

Multi-shot techniques, on the other hand, offer highresolution DWI by effectively suppressing image distortions. However, they can introduce strong ghost artifact if data are reconstructed directly because of phase variations among different shots [7]. By using phase correction, which is conducted through either extra navigator or self-navigator, the aforementioned artifact can be minimized, and subsequently improves image quality [8-10]. Since the reconstruction with phase correction is basically based on parallel imaging principles, extra navigator is usually needed for high shot numbers such as 6 shots in order to maintain a reliable performance. The multi-shot DWI techniques, including interleaved EPI or readout-segmented EPI [11], have been applied in brain and other body organs with promising results [12-16].

Navigated interleaved multi-shot echo-planar imaging (IMS-EPI) is more effective in distortion reduction compared with readout-segmented EPI [11]. In navigated IMS-EPI, a kspace domain reconstruction method, GRAPPA with a compact kernel is used to recover missing data in each shot, which has been shown to be more robust than image domain phase correction method [10].

Herein, the aims of our study were to compare the image quality and assess the ADC, signal-to-noise ratio (SNR), contrast-to-noise ratio (CNR), and geometric distortion between IMS-EPI and SS-EPI in female pelvis.

\section{Materials and methods}

\section{Study information}

This was a prospective study approved by the local ethics committee with written informed consent from participating subjects. Consecutive females who underwent pelvic MRI in our unit were prospectively recruited between January 2016 and September 2017. Inclusion criteria were (1) females with gynecological symptoms (heavy flow, abnormal bleeding, irregular menses, and dysmenorrhea, etc.); (2) clinical- or ultrasound-suspected uterine congenital anomalies; (3) ultrasound-detected indeterminate masses in the pelvis or with raised CA125; and (4) pre-operative or post-operative assessment of histologic-proven gynecological cancers. Exclusion criteria were those with (1) any contraindications to MRI; (2) hip prosthesis; and (3) no DWI performed.

\section{MRI technique}

All MRI examinations were acquired on a $3 \mathrm{~T}$ MRI (Achieva 3.0T TX, Philips Healthcare) using a 16channel phased-array torso coil. All the patients fasted for $6 \mathrm{~h}$ and received $20 \mathrm{mg}$ intravenous hyoscine butylbromide (Buscopan, Boehringer Ingelheim) to reduce the peristaltic artifacts. Standard abdominopelvic MRI was performed with the scanning parameters summarized in Table 1. The axial T2-weighted (T2W) images and DWI images had the exact same anatomical coverage, slice thickness, and interslice gap to ensure image registration for subsequent analysis.

SS-EPI and IMS-EPI sequences were acquired using $3 b$ values $\left(b=0,400,800 \mathrm{~s} / \mathrm{mm}^{2}\right)$ based on the same anatomical coverage. The choice of the highest $b$ value in this study was based on a balance between sufficient signal suppression of normal tissues in the female pelvis and scan time [17]. The acquisition time for SS-EPI and IMS-EPI was $2.00 \mathrm{~min}$ and $6.83 \mathrm{~min}$, respectively. IMS-EPI was acquired using a multishot DWI sequence (number of shots $=4$ ), with a partial Fourier factor of 0.76 . To be noted, a low-resolution fully sampled navigator was acquired after the image data in each shot for IMS-EPI, which was used for monitoring phase variations and then phase correction in the image reconstruction. 
Table 1 Summary of MRI scanning parameters

\begin{tabular}{lllllll}
\hline Sequences & Sagittal T2WI & Coronal T2WI & Axial T2WI & SS-EPI & IMS-EPI & CE 3D T1WI \\
\hline Pulse & Free-breathing & Free-breathing & Free-breathing & Free-breathing & Free-breathing & Free-breathing \\
TR/TE (ms) & $4654 / 80$ & $3000 / 80$ & $2800 / 100$ & $3240 / 57$ & $4237 / 50$ & $3.1 / 1.45$ \\
FOV $\left(\mathrm{mm}^{2}\right)$ & $240 \times 240$ & $240 \times 378$ & $240 \times 371$ & $350 \times 290$ & $300 \times 200$ & $370 \times 250$ \\
Matrix size & $480 \times 300$ & $160 \times 220$ & $344 \times 507$ & $160 \times 129$ & $200 \times 132$ & $248 \times 166$ \\
Number of directions & N.A. & N.A. & N.A. & 3 & 3 & 2 \\
Number of averages & 2 & 1 & 1 & 6 & 4 & 1 \\
Slice thickness (mm) & 4 & 5 & 4 & 15.156 & 14.902 & 3 \\
WFS (pix)/BW (Hz) & 2.002 & 1.033 & 2.845 & 6.90 & 2.00 & 6.83 \\
Acquisition time (min) & 3.48 & 2.00 & & & 0.32 \\
\hline
\end{tabular}

$C E$, contrast-enhanced; TR/TE, repetition time/echo; FOV, field of view; N.A., not applicable

\section{IMS-EPI reconstruction}

The image reconstruction was performed in Matlab R2018b (The MathWorks, Inc.). Reconstruction was conducted in the $\mathrm{k}$-space domain using GRAPPA-like interpolation to recover missing data in each shot. Phase variation, which was induced by physiological motion during diffusion gradient encoding, was used for signal encoding, analogy to coil sensitivity encoding. The GRAPPA weights were calibrated from the navigator and applied to the image-echo $\mathrm{k}$-space to recover the data of each channel and shot. The reconstruction method was summarized in Fig. 1. Full details of the reconstruction method were discussed in previous work [10].

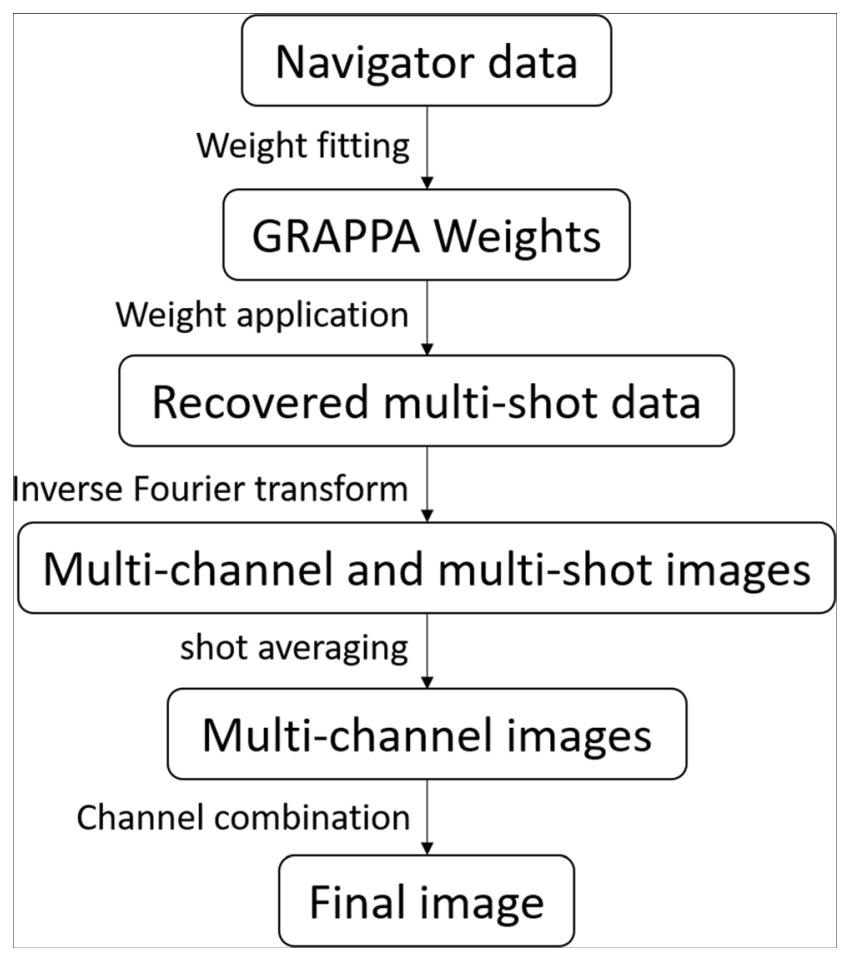

Fig. 1 Interleaved multi-shot echo-planar imaging (IMS-EPI) reconstruction method

\section{Qualitative assessment}

The image quality was assessed on Image $\mathrm{J}$ viewing platform $1.45 \mathrm{~s}$ freeware (National Institutes of Health) by two reviewers (radiologist 1 with 3 years cross-sectional imaging experience; radiologist 2 with more than 10 years crosssectional and pelvic MRI imaging experience) on separate reading sessions providing independent evaluations. DWI sequences and patients' sequences were randomly allocated, so the reviewers were blinded to the type of DWI sequences and patient's clinical information. Qualitative visual assessment was performed on the $b=800 \mathrm{~s} / \mathrm{mm}^{2}$ images and based on a 5-point Likert scale on overall image quality, artifacts, and sharpness. Sharpness was defined by the clarity of the boundaries of the uterus. In patients with an identifiable lesion in the pelvis and without history of pelvic surgery, lesion conspicuity was also assessed (Table 2).

\section{Quantitative assessment}

\section{ADC}

Patients with history of hysterectomy were excluded from the quantitative assessment. ADC maps were generated from both DWI sequences with a mono-exponential fit based on the acquired $3 b$ values using in-house scripts written on MATLAB.

Radiologist 1 placed two sets of regions of interest (ROIs) on $b=800 \mathrm{~s} / \mathrm{mm}^{2}$ SS-EPI and IMS-EPI (Fig. 2) and then transferred to the corresponding ADC maps with reference to the $\mathrm{T} 2 \mathrm{~W}$ images. ROI 1 was placed in the endometrium on the slice with the largest diameter and ROI $2(1 \mathrm{~cm} \times 1 \mathrm{~cm})$ was placed in the gluteus muscles (GM) on the same slice to quantify the $\mathrm{ADC}$ values.

\section{Signal-to-noise ratio and contrast-to-noise ratio}

The aforementioned ROIs were also transferred to $b=0$ and $400 \mathrm{~s} / \mathrm{mm}^{2}$ images. The average signal within the ROIs in the 
Table 2 Image assessment based on the 5-point Likert scale

\begin{tabular}{|c|c|c|c|c|}
\hline Score & Overall image quality & Artifacts & Sharpness & Lesion conspicuity \\
\hline 1 & Non-diagnostic & Non-diagnostic & Non-diagnostic & Lesion unidentifiable \\
\hline 2 & Substantial deficits in image quality & Substantial impact on diagnosis & Not sharp & $\begin{array}{l}\text { No differentiation between lesion and } \\
\text { normal anatomy }\end{array}$ \\
\hline 3 & Moderate image quality & Moderate impact on diagnosis & A little sharp & Subtle lesion with poorly defined edges \\
\hline 4 & Good image quality & Little impact on image diagnosis & Moderately sharp & Well-seen lesion with poorly defined edges \\
\hline 5 & Excellent image quality & No artifact & Satisfying sharp & Well-seen lesion with well-defined edges \\
\hline
\end{tabular}

endometrium and GM on different $b$ values was denoted as $\mathrm{S}_{\mathrm{ENDO}}, \mathrm{S}_{\mathrm{ENDO} 400}, \mathrm{~S}_{\mathrm{ENDO} 800}, \mathrm{~S}_{\mathrm{GM} 0}, \mathrm{~S}_{\mathrm{GM} 400}$, and $\mathrm{S}_{\mathrm{GM} 800}$, respectively. Signal-to-noise ratio (SNR) was defined as the signal of endometrium divided by the standard deviation [18]:

$\mathrm{SNR}=\mathrm{S}_{E N D O} / \mathrm{SD}_{E N D O}$

Contrast-to-noise ratio (CNR) was defined as the absolute signal difference of endometrium and GM divided by the standard deviation of GM [18]:

$\mathrm{CNR}=\left|\mathrm{S}_{E N D O}-\mathrm{S}_{\mathrm{GM}}\right| / \mathrm{SD}_{\mathrm{GM}}$

\section{Geometric distortion}

This was assessed by measuring the deviations in maximal diameter of the uterus in transverse and anterior-posterior directions between the two DWI sequences on the slice that the uterus appeared largest. The measurements on T2W images on the correlated slice were taken as standard of reference.

\section{Statistical analysis}

Inter-rater agreement for qualitative image quality was assessed using $\kappa$ statistics ( $<0$, poor; 0.01-0.20, slight; 0.21-0.40, fair; $0.41-0.60$, moderate; $0.61-0.80$, good; $0.81-0.99$, almost perfect) [19]. The correlation between the reviewers' scores was determined by Kendall test. Qualitative scores were compared using Wilcoxon signed-rank test; the mean ADC, SNR, CNR, and geometric distortion between
SS-EPI and IMS-EPI were compared using paired $t$ test and Bland-Altman analysis after testing for normality. All statistical analyses were performed using SPSS software (version 22.0, SPSS Inc.). $P<0.05$ was considered as statistically significant.

\section{Results}

\section{Demographics}

Eighty patients (mean age 53.9, range $23-86$ years old) were included in the qualitative assessment. The indications for pelvic MRI included (1) for investigation of gynecological symptoms (heavy flow, abnormal bleeding, irregular menses, and dysmenorrhea etc.) $(n=29)$; (2) clinical- or ultrasoundsuspected uterine congenital anomalies $(n=2)$; (3) ultrasounddetected indeterminate masses in the pelvis or with raised CA125 ( $n=15$ ); and (4) pre-operative or post-operative assessment of histologic-proven gynecological cancers $(n=34)$.

Twelve patients had hysterectomy previously, thus were excluded from subsequent quantitative analysis. Among the 68 patients who were included in the quantitative analysis, there were endometrial cancer $(n=37)$, benign diseases (uterine fibroid, $n=12$; adenomyosis, $n=3$; ovarian teratoma; $n=$ $2)$, uterine congenital anomalies $(n=2)$, other gynecological malignancies (cervix carcinoma, $n=3$; ovarian carcinoma, $n=3$; carcinoma of vulva, $n=2$ ), and 4 cases with no structural abnormality found.
Fig. 2 ROIs on the (1) endometrium and (2) gluteus muscles on $b=800 \mathrm{~s} / \mathrm{mm}^{2}$ images SS-EPI (a) and IMS-EPI (b)

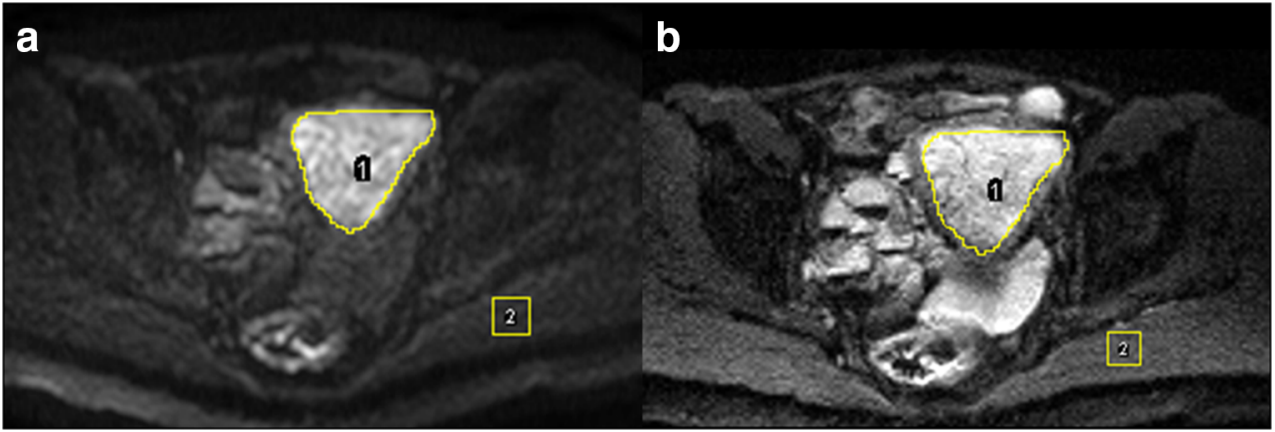




\section{Qualitative assessment}

IMS-EPI scored higher image quality than SS-EPI on all the qualitative factors evaluated regardless of the experience of reviewers (Figs. 3, 4, and 5; Table 3). The average scores between the reviewers for SS-EPI vs. IMS-EPI were as follows: overall quality 3.04 vs. 4.17 , artifacts 3.09 vs. 3.99 , sharpness 2.40 vs. 4.32 , and lesion conspicuity 3.20 vs. 4.25 $(p<0.001)$.

The median $\kappa$ scores between the two reviewers were high: SS-EPI 0.699 (95\% confidence interval (CI), 0.630-0.768) and IMS-EPI 0.702 (95\% CI, 0.631-0.773) [19]. The average Kendall $r$ correlations were significantly positive (SS-EPI: $r=0.742, p<0.001$; IMS-EPI: $r=0.789, p<0.001$ ).

\section{Quantitative assessment}

The average ADCs of endometrium were $(1.307 \pm 0.354) \times$ $10^{-3} \mathrm{~mm}^{2} / \mathrm{s}$ on SS-EPI and $(1.214 \pm 0.348) \times 10^{-3} \mathrm{~mm}^{2} / \mathrm{s}$ on IMS-EPI $(p=0.063)$. The average ADCs of GM were $(1.348$ $\pm 0.454) \times 10^{-3} \mathrm{~mm}^{2} / \mathrm{s}$ on SS-EPI and $(1.349 \pm 0.343) \times 10^{-3}$ $\mathrm{mm}^{2} / \mathrm{s}$ on IMS-EPI $(p=0.976)$ (Fig. 6).

The average SNR of SS-EPI was $5.305 \pm 1.803,5.695 \pm$ 2.213 , and $5.465 \pm 1.978$ in comparison to that of $4.539 \pm$ $1.693,4.752 \pm 1.915$, and $5.004 \pm 2.198$ on IMS-EPI on $b=$ 0,400 , and $800 \mathrm{~s} / \mathrm{mm}^{2}(p<0.001, p<0.001$, and $p=0.030)$, respectively. The average CNR of SS-EPI was $11.406 \pm$ $6.205,13.816 \pm 7.134$, and $14.122 \pm 8.613$ in comparison to that of $7.414 \pm 8.622,6.504 \pm 6.058$, and $7.183 \pm 5.331$ on IMS-EPI on $b=0,400$, and $800 \mathrm{~s} / \mathrm{mm}^{2}(p<0.001, p<0.001$, and $p<0.001)$, respectively.
The average geometric distortion on SS-EPI was $4.093 \pm$ $3.336 \mathrm{~mm}$ (transverse direction) and $3.062 \pm 3.680 \mathrm{~mm}$ (anterior-posterior direction) and on IMS-EPI was $3.180 \pm$ $2.306 \mathrm{~mm}$ (transverse direction) and $2.377 \pm 2.068 \mathrm{~mm}$ (anterior-posterior direction) ( $p=0.044,0.018)$, respectively.

\section{Discussion}

In this study, we showed that IMS-EPI had superior image quality and decrease geometric distortion compared with SSEPI without affecting ADC quantification. However, the SNR and CNR suffered, being lower on IMS-EPI.

Traditionally, DWI based on SS-EPI allows rapid acquisition but unfortunately is more susceptible to artifacts, such as chemical shift, Nyquist ghost, image blurring, and geometric distortion. By lowering FOV in the phase-encoding direction of the EPI read-out, the off-resonance-induced artifact could be minimized and image quality could be improved [20]. With zoomed EPI, instead of standard EPI pulse, the distortion and ghosting artifacts can be reduced. [21, 22] However, for patients with disseminate diseases, a reduced FOV cannot fulfill the clinical need for whole pelvic evaluation. Moreover, the mean tumor ADC obtained with zoomed EPI is not stable [23-25].

The image quality was improved on IMS-EPI with superior overall image quality, less artifacts, increased sharpness of the image, and higher lesion conspicuity when compared with SSEPI. The findings were consistent between reviewers, regardless of their experience with substantial inter-rater agreement. In comparison with SS-EPI, IMS-EPI has higher bandwidth in phase-encoding direction, which can reduce distortion and

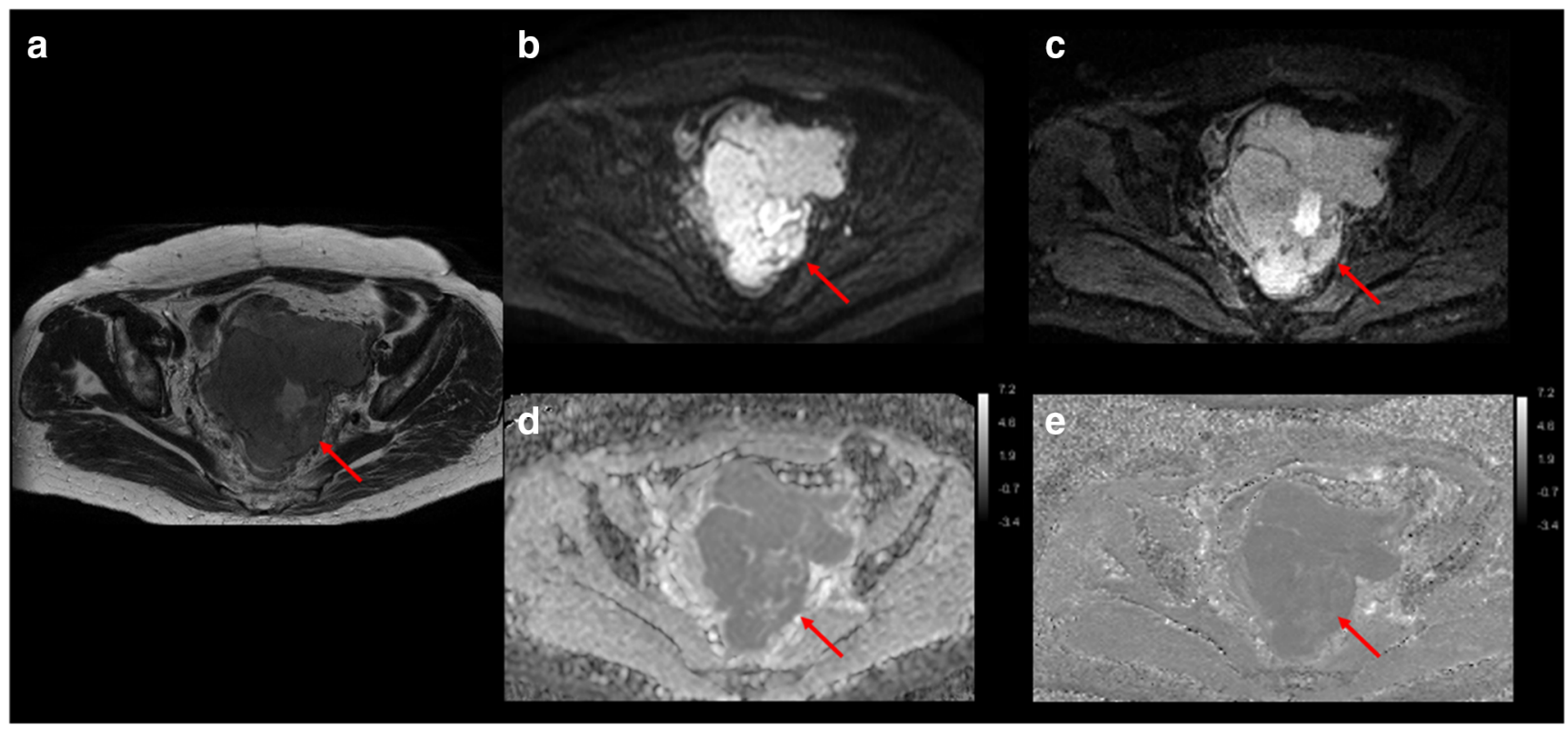

Fig. 3 An example of single-shot k-space trajectory echo-planar imaging (SS-EPI) and interleaved multi-shot echo-planar imaging (IMS-EPI) of a patient with endometrial cancer (red arrow). a Axial T2WI. b SS-EPI ( $b=$ $\left.800 \mathrm{~s} / \mathrm{mm}^{2}\right)$. c IMS-EPI $\left(b=800 \mathrm{~s} / \mathrm{mm}^{2}\right)$. d ADC map for SS-EPI. e ADC map for IMS-EPI 


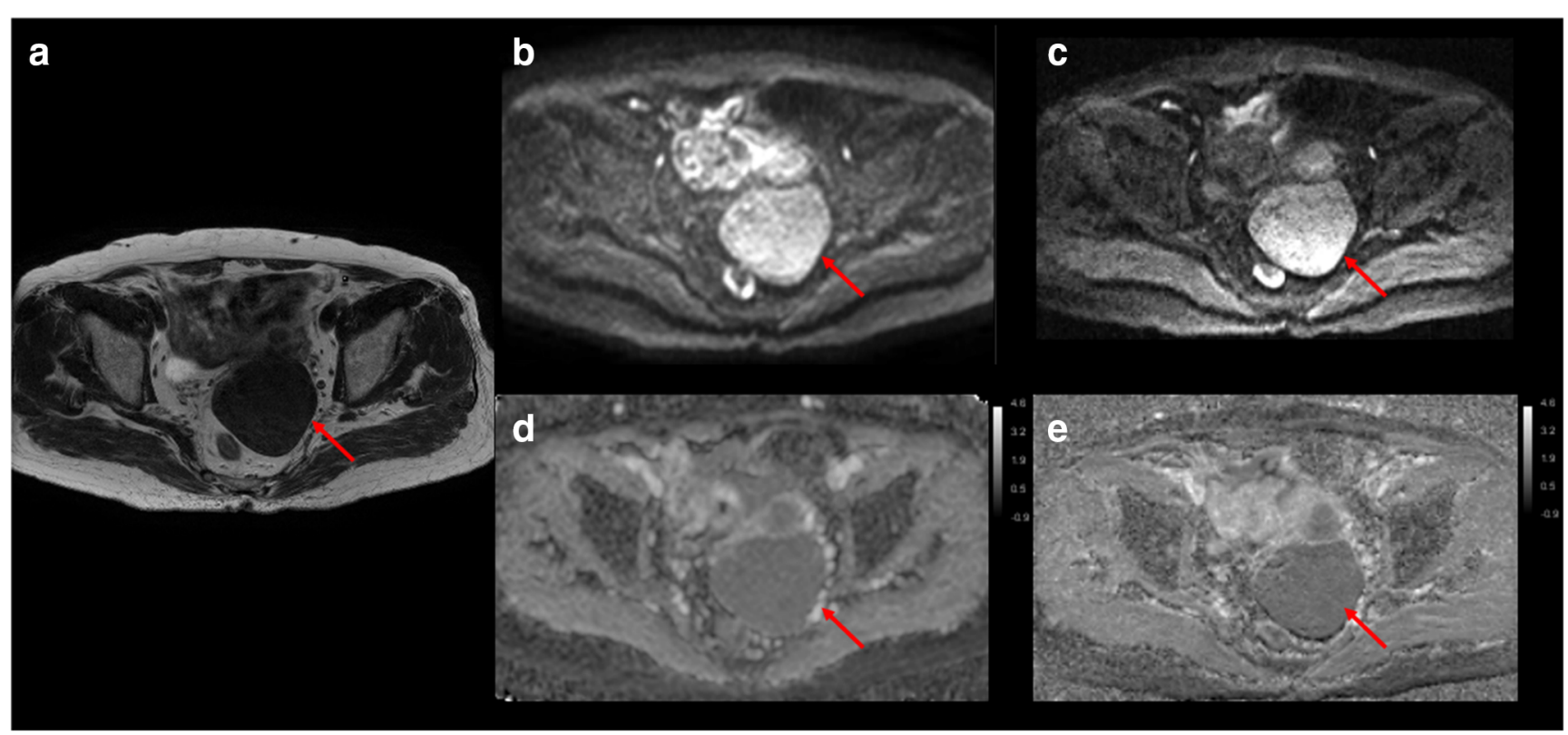

Fig. 4 An example of single-shot k-space trajectory echo-planar imaging (SS-EPI) and interleaved multi-shot echo-planar imaging (IMS-EPI) of a patient with leiomyoma (red arrow). a Axial T2WI. b SS-EPI ( $b=800$

improve spatial resolution, thus providing higher fidelity for the image details, explaining the improved image quality on IMS-EPI.

The ADC has been shown to be a strong predictor for histologic subtype and tumor grade in endometrial cancer [26] but can be influenced by magnetic field strength, sequence protocols, and the $b$ values used [27]. The novel reconstruction method used by IMS-EPI did not affect the quantification of ADC. In other words, quantification of IMS-EPI is at least as robust as SS-EPI with benefit of better image quality. This is important as $\left.\mathrm{s} / \mathrm{mm}^{2}\right)$. c IMS-EPI $\left(b=800 \mathrm{~s} / \mathrm{mm}^{2}\right)$. d ADC map for SS-EPI. e ADC map for IMS-EPI

the absolute value of ADC can be used to differentiate malignant endometrial lesion from normal endometrium [28]. Furthermore, the change in ADC can monitor treatment response and it is imperative that the derivation of ADC is reproducible, in order to trace the real therapeutic magnitude [29].

Usually, normal myometrium was taken as reference when assessing CNR in female pelvis [30], but in our study, many of the patients with endometrial cancer had bulky tumors and the identification of normal myometrium was inconsistent and

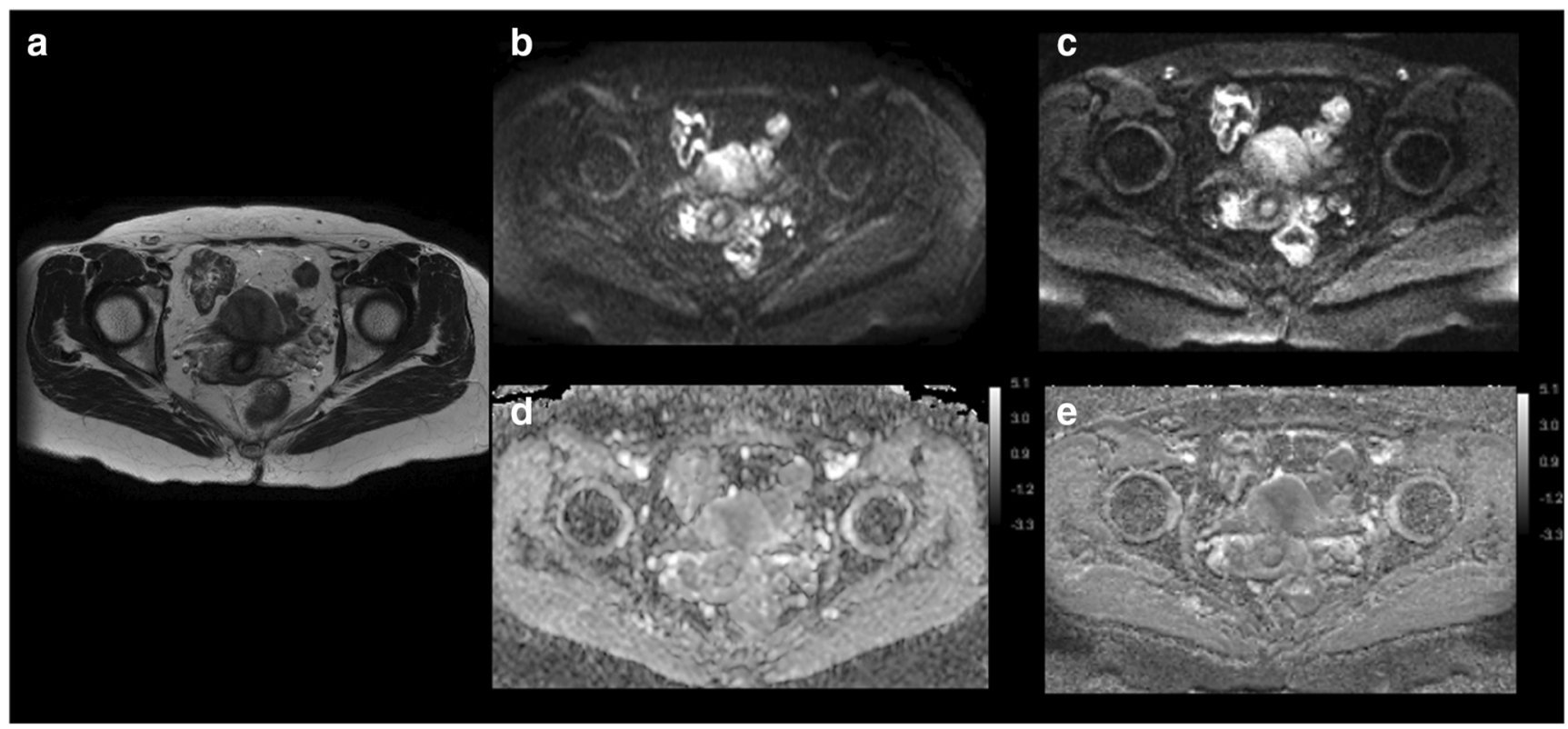

Fig. 5 An example of single-shot k-space trajectory echo-planar imaging (SS-EPI) and interleaved multi-shot echo-planar imaging (IMS-EPI) of patient without gynecological abnormality. a Axial T2WI. b SS-EPI $(b=$ $\left.800 \mathrm{~s} / \mathrm{mm}^{2}\right)$. c IMS-EPI $\left(b=800 \mathrm{~s} / \mathrm{mm}^{2}\right)$. d ADC map for SS-EPI. e ADC map for IMS-EPI 
Table 3 Qualitative scores between the two reviewers

\begin{tabular}{lcccc}
\hline & Overall image & Artifacts & Sharpness & $\begin{array}{l}\text { Lesion } \\
\text { conspicuity }\end{array}$ \\
\hline$N$ & 80 & 80 & 68 & 62 \\
Reader 1 & & & & \\
SS-EPI & 3.15 & 3.34 & 2.41 & 3.13 \\
IMS-EPI & 4.20 & 4.09 & 4.29 & 4.24 \\
$p$ & $<0.001$ & $<0.001$ & $<0.001$ & $<0.001$ \\
Reader 2 & & & & \\
SS-EPI & 2.94 & 2.98 & 2.40 & 3.29 \\
IMS-EPI & 4.13 & 3.95 & 4.34 & 4.26 \\
$p$ & $<0.001$ & $<0.001$ & $<0.001$ & $<0.001$ \\
\hline
\end{tabular}

SS-EPI, single-shot k-space trajectory echo-planar imaging (SS-EPI); IMS-EPI, multi-shot echo-planar imaging

unreliable. For some patients with diffuse adenomyosis, the identification of normal myometrium on the same slice as the endometrium ROI was also challenging. As such, we had chosen to take GM signal as an alternative reference [18].

We observed lower SNR and CNR on IMS-EPI than SS-EPI, which could be a result from the differences in spatial resolution and post-processing method. Given that IMS-EPI offered higher spatial resolution, the SNR would decrease unless more averages were used. Nevertheless, increasing averages would incur penalty in the scan time and make this unpractical for clinical use. Therefore, in this study, we elected to use two as a trade-off between SNR and scan time. The CNR drop in IMS-EPI is probably caused by the enhanced signal intensity in the GM (Fig. 2) due to coil sensitivities, but we were not able to conduct the uniformity correction like traditional DWI since the IMSEPI was reconstructed offline. Furthermore, the differences in imaging parameters of both sequences could account for the signal differences in the tissues investigated.

Nevertheless, despite lower SNR and CNR, the overall image quality was higher on IMS-EPI, likely attributed to the significant improvement in geometric distortion on IMS-EPI and hence confidence in defining anatomical borders. Studies have shown that DWI coupled with T2W images can improve the evaluation of various gynecological cancers. A prediction model was constructed combining both sequences to evaluate parametrial invasion (PMI) in cervical cancer [31]; DWI significantly increases the specificity of MR imaging in the detection of residual tumor compared with $\mathrm{T} 2 \mathrm{~W}$ images alone in cervical cancer after radiotherapy $[32,33]$. For endometrial cancer, the depth of myometrial invasion and the presence of lymph node metastasis are important prognostic factors. DWI coupled with $\mathrm{T} 2 \mathrm{~W}$ images can offer high diagnostic performance with an area under the receiver operating characteristic (ROC) curve of 0.94 in predicting myometrial invasion [34]. The reduction in geometric distortion on IMS-EPI would benefit the coregistration between the DWI and anatomical images and potentially allow more accurate assessment, better surgical planning and treatment stratification.

However, the current longer scan time incurred in IMS-EPI would limit its clinical utility but other techniques could be considered to improve the acquisition efficiency, for example, the application of simultaneous multi-slice technique, which could shorten scan time by a factor of 2-3 without losing significant SNR [12, 35]. Furthermore, the development of an on-line image reconstruction would also improve the clinical acceptance of this promising technique.

Our study has limitations. First, we had a heterogeneous cohort of patients with various pelvic conditions including

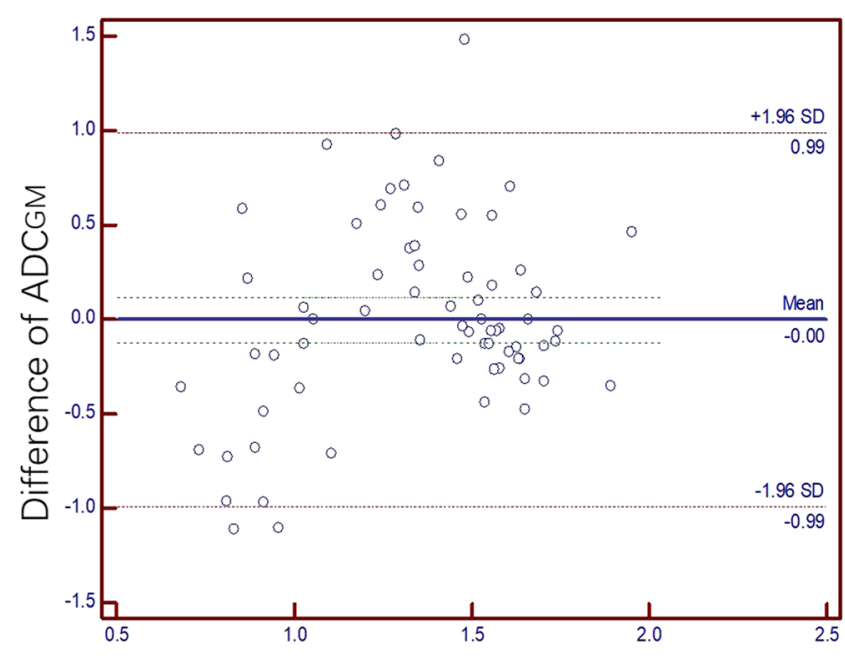

Average of ADCGM on SS-EPI and IMS-EPI

Average of ADCENDO on SS-EPI and IMS-EPI

Fig. 6 Bland-Altman plots comparing the ADC values between single-shot k-space trajectory echo-planar imaging (SS-EPI) DWI and interleaved multishot echo-planar imaging (IMS-EPI) DWI. $\mathrm{ADC}_{\mathrm{GM}}$ : the average $\mathrm{ADCs}$ of gluteus muscle; $\mathrm{ADC}_{\mathrm{ENDO}}$ : the average $\mathrm{ADCs}$ of endometrium 
both malignant and benign diseases; thus, the merits of IMSEPI in assisting the diagnosis of specific disease such as endometrial cancer could not be evaluated. Second, the inherent longer scan time required by IMS-EPI and offline image reconstruction may limit its clinical translation currently. Continual effort is needed to improve the efficiency of data acquisition and a more streamline post-processing algorithm to minimize these hindrances of a promising technique. For example, simultaneous multi-slice technique can be used to accelerate IMS-EPI DWI $[35,36]$, with a smaller decrease of SNR compared with traditional parallel imaging techniques.

In conclusion, IMS-EPI showed higher image quality and lower geometric distortion compared with SS-EPI without affecting the mean ADC, potentially a promising technique in improving assessment in female pelvis. However, the SNR and CNR suffered due to the post-processing limitations.

Funding information The authors state that this work has not received any funding.

\section{Compliance with ethical standards}

Guarantor The scientific guarantor of this publication is Elaine Yuen Phin Lee.

Conflict of interest The authors declare that they have no conflict of interest.

Statistics and biometry No complex statistical methods were necessary for this paper.

Informed consent Written informed consent was obtained from all subjects (patients) in this study by the Department of Diagnostic Radiology, HKU.

Ethical approval Institutional Review Board approval was obtained (HKU/HA HKW IRB UW 17-404).

\section{Methodology}

- Prospective

- Observational

- Performed at one institution

Open Access This article is distributed under the terms of the Creative Commons Attribution 4.0 International License (http:// creativecommons.org/licenses/by/4.0/), which permits unrestricted use, distribution, and reproduction in any medium, provided you give appropriate credit to the original author(s) and the source, provide a link to the Creative Commons license, and indicate if changes were made.

\section{References}

1. Bakir B, Sanli S, Bakir VL et al (2017) Role of diffusion weighted MRI in the differential diagnosis of endometrial cancer, polyp, hyperplasia, and physiological thickening. Clin Imaging 41:86-94
2. Angioli R, Plotti F, Capriglione S et al (2016) Preoperative local staging of endometrial cancer: the challenge of imaging techniques and serum biomarkers. Arch Gynecol Obstet 294:1291-1298

3. Manoharan D, Das CJ, Aggarwal A, Gupta AK (2016) Diffusion weighted imaging in gynecological malignancies - present and future. World J Radiol 8:288-297

4. Dietrich O, Biffar A, Baur-Melnyk A, Reiser MF (2010) Technical aspects of MR diffusion imaging of the body. Eur J Radiol 76:314 322

5. Zhang Z, Huang F, Ma X, Xie S, Guo H (2015) Self-feeding MUSE: a robust method for high resolution diffusion imaging using interleaved EPI. Neuroimage 105:552-560

6. Barentsz MW, Taviani V, Chang JM et al (2015) Assessment of tumor morphology on diffusion-weighted (DWI) breast MRI: diagnostic value of reduced field of view DWI. J Magn Reson Imaging 42:1656-1665

7. Peng Y, Li Z, Tang H et al (2018) Comparison of reduced field-ofview diffusion-weighted imaging (DWI) and conventional DWI techniques in the assessment of rectal carcinoma at 3.0T: image quality and histological T staging. J Magn Reson Imaging 47: 967-975

8. Xie VB, Lyu M, Wu EX (2017) EPI Nyquist ghost and geometric distortion correction by two-frame phase labeling. Magn Reson Med 77:1749-1761

9. Chang HC, Hui ES, Chiu PW, Liu X, Chen NK (2018) Phase correction for three-dimensional (3D) diffusion-weighted interleaved EPI using 3D multiplexed sensitivity encoding and reconstruction (3D-MUSER). Magn Reson Med 79:2702-2712

10. Ma X, Zhang Z, Dai E, Guo H (2016) Improved multi-shot diffusion imaging using GRAPPA with a compact kernel. Neuroimage 138:88-99

11. Wang Y, Ma X, Zhang Z et al (2018) A comparison of readout segmented EPI and interleaved EPI in high-resolution diffusion weighted imaging. Magn Reson Imaging 47:39-47

12. Dai E, Ma X, Zhang Z, Yuan C, Guo H (2017) Simultaneous multislice accelerated interleaved EPI DWI using generalized blippedCAIPI acquisition and 3D K-space reconstruction. Magn Reson Med 77:1593-1605

13. Hu J, Li M, Dai Y et al (2018) Combining SENSE and reduced field-of-view for high-resolution diffusion weighted magnetic resonance imaging. Biomed Eng Online 17:77

14. van Rijssel MJ, Zijlstra F, Seevinck PR et al (2019) Reducing distortions in echo-planar breast imaging at ultrahigh field with highresolution off-resonance maps. Magn Reson Med. https://doi.org/ $10.1002 / \mathrm{mrm} .27701$

15. Li L, Wang L, Deng M et al (2015) Feasibility study of 3-T DWI of the prostate: readout-segmented versus single-shot echo-planar imaging. AJR Am J Roentgenol 205:70-76

16. Ihalainen T, Kuusela L, Soikkeli M, Lantto E, Ovissi A, Sipila O (2016) A body-sized phantom for evaluation of diffusion-weighted MRI data using conventional, readout-segmented, and zoomed echo-planar sequences. Acta Radiol 57:947-954

17. Forstner R, Thomassin-Naggara I, Cunha TM et al (2017) ESUR recommendations for MR imaging of the sonographically indeterminate adnexal mass: an update. Eur Radiol 27:2248-2257

18. Panyarak W, Chikui T, Yamashita Y, Kamitani T, Yoshiura K (2018) Image quality and ADC assessment in turbo spin-echo and echo-planar diffusion-weighted MR imaging of tumors of the head and neck. Acad Radiol. https://doi.org/10.1016/j.acra.2018.11.016

19. Viera AJ, Garrett JM (2005) Understanding interobserver agreement: the kappa statistic. Fam Med 37:360-363

20. Sohaib SA, Sahdev A, Van Trappen P, Jacobs IJ, Reznek RH (2003) Characterization of adnexal mass lesions on MR imaging. AJR Am J Roentgenol 180:1297-1304

21. Rosenkrantz AB, Chandarana H, Pfeuffer J et al (2015) Zoomed echo-planar imaging using parallel transmission: impact on image 
quality of diffusion-weighted imaging of the prostate at $3 \mathrm{~T}$. Abdom Imaging 40:120-126

22. Ota T, Hori M, Onishi $\mathrm{H}$ et al (2017) Preoperative staging of endometrial cancer using reduced field-of-view diffusion-weighted imaging: a preliminary study. Eur Radiol 27:5225-5235

23. Kim H, Lee JM, Yoon JH et al (2015) Reduced field-of-view diffusion-weighted magnetic resonance imaging of the pancreas: comparison with conventional single-shot echo-planar imaging. Korean J Radiol 16:1216-1225

24. Lu Y, Hatzoglou V, Banerjee $\mathrm{S}$ et al (2015) Repeatability investigation of reduced field-of-view diffusion-weighted magnetic resonance imaging on thyroid glands. J Comput Assist Tomogr 39: 334-339

25. Feng Z, Min X, Sah VK et al (2015) Comparison of field-of-view (FOV) optimized and constrained undistorted single shot (FOCUS) with conventional DWI for the evaluation of prostate cancer. Clin Imaging 39:851-855

26. Tanaka T, Terai Y, Fujiwara S et al (2018) Preoperative diffusionweighted magnetic resonance imaging and intraoperative frozen sections for predicting the tumor grade in endometrioid endometrial cancer. Oncotarget 9:36575-36584

27. Dong H, Li Y, Li H, Wang B, Hu B (2014) Study of the reduced field-of-view diffusion-weighted imaging of the breast. Clin Breast Cancer 14:265-271

28. Rechichi G, Galimberti S, Signorelli M et al (2011) Endometrial cancer: correlation of apparent diffusion coefficient with tumor grade, depth of myometrial invasion, and presence of lymph node metastases. AJR Am J Roentgenol 197:256-262

29. Padhani AR, Liu G, Koh DM et al (2009) Diffusion-weighted magnetic resonance imaging as a cancer biomarker: consensus and recommendations. Neoplasia 11:102-125

30. Utsunomiya D, Notsute S, Hayashida Y et al (2004) Endometrial carcinoma in adenomyosis: assessment of myometrial invasion on
T2-weighted spin-echo and gadolinium-enhanced T1-weighted images. AJR Am J Roentgenol 182:399-404

31. Park JJ, Kim CK, Park SY, Park BK, Kim B (2014) Value of diffusion-weighted imaging in predicting parametrial invasion in stage IA2-IIA cervical cancer. Eur Radiol 24:1081-1088

32. Thomeer MG, Vandecaveye V, Braun L et al (2019) Evaluation of T2-W MR imaging and diffusion-weighted imaging for the early post-treatment local response assessment of patients treated conservatively for cervical cancer: a multicentre study. Eur Radiol 29: 309-318

33. Jalaguier-Coudray A, Villard-Mahjoub R, Delouche A et al (2017) Value of dynamic contrast-enhanced and diffusion-weighted MR imaging in the detection of pathologic complete response in cervical cancer after neoadjuvant therapy: a retrospective observational study. Radiology 284:432-442

34. Deng L, Wang QP, Chen X, Duan XY, Wang W, Guo YM (2015) The combination of diffusion- and $\mathrm{T} 2$-weighted imaging in predicting deep myometrial invasion of endometrial cancer: a systematic review and meta-analysis. J Comput Assist Tomogr 39: 661-673

35. Setsompop K, Gagoski BA, Polimeni JR, Witzel T, Wedeen VJ, Wald LL (2012) Blipped-controlled aliasing in parallel imaging for simultaneous multislice echo planar imaging with reduced g-factor penalty. Magn Reson Med 67:1210-1224

36. Dai E, Zhang Z, Ma X et al (2018) The effects of navigator distortion and noise level on interleaved EPI DWI reconstruction: a comparison between image- and k-space-based method. Magn Reson Med 80:2024-2032

Publisher's note Springer Nature remains neutral with regard to jurisdictional claims in published maps and institutional affiliations. 